\title{
Effectiveness of Radial Shockwave Therapy in Calcific and Non-Calcific Tendinopathy of the Shoulder: a Systematic Review and Meta-Analysis
}

\author{
A. Ammendolia ${ }^{1}$, N. Marotta ${ }^{1}$, A. Demeco ${ }^{1}$, C. Marinaro' ${ }^{1}$, L. Moggio ${ }^{1}$, \\ M. Barletta ${ }^{1}$, C. Costantino ${ }^{2}$ \\ 1 Department of Surgical and Medical Sciences, University of Catanzaro "Magna Graecia", Catanzaro Italy \\ 2 Department of Medicine and Surgery, University of Parma, Parma, Italy
}

\section{CORRESPONDING AUTHOR:}

Nicola Marotta

Via Eraclea 7

88100, Catanzaro, Italy

Phone: +393201414038

Fax: +390961712812

E-mail: nicolakr@gmail.com

DOI:

10.32098/mltj.01.2020.05

LEVEL OF EVIDENCE: $1 a$

\begin{abstract}
SUMMARY
Objective. To investigate RCTs that reported the effects of Radial ShockWave Therapy (RSWT) versus traditional physiotherapy on pain, shoulder articular function and quality of life of patients suffering by tendinopathy of the shoulder.

Data sources. Studies were comprehensively searched, without language restrictions, on PubMed, Embase, PEdro, Cochrane Controlled Trials Register, the Cochrane. Possible studies from reference lists of articles and review were cross-checked.

Study selection. Randomized controlled trials from 2006 to present were included. Two reviewers conducted the selection independently.

Data extraction. Two reviewers assessed the quality of data. Disagreements in extraction were settled by consulting a third and fourth reviewers to reach a consensus.

Data synthesis. Five studies with 475 participants were included in the network meta-analysis that used a random-effect model to investigate the mean difference of pooled effect sizes of the visual analog scale, Constant-Murley score combined with UCLA, and SPADI score.

Conclusions. Compared to control groups, RSWT resulted an improvement in pain -0.24 (95\% CI: -0.43 to $-0.05 \mathrm{Z}=2.45 \mathrm{p}<0.05$ ), an increase of Constant/UCLA score 4.72 (95\% CI: 4.03 to $5.4 \mathrm{Z}=13.5 \mathrm{p}<0.05)$ and a decrease in SPADI scale -6.76 (95\% CI: -13.3 to $-0.48 \mathrm{Z}=2.11$ $\mathrm{p}<0.05$ ). Despite the small number of the studies, seems that RSWT can be more effective in pain, shoulder articular function and quality of life of patients with tendinopathy of the shoulder.
\end{abstract}

\section{KEY WORDS}

Radial Shockwave Therapy; shoulder tendinitis; calcific shoulder tendinopathy; non-calcific shoulder tendinopathy

\section{INTRODUCTION}

Shoulder pain due to rotator cuff (RC) tendinitis is one of the most prevalent and costly work-related musculoskeletal disorders. 1 In $7 \%$ to $17 \%$ of cases, shoulder pain is characterized by inflammation and associated with a calcification in the rotator cuff, hence the term calcific RC tendinitis $(1,2)$.

The clinical symptoms are pain, decreased range of motion and progressive disability of the shoulder. The initial treatments are conservative and include physiotherapy and nonsteroidal anti-inflammation steroidal drugs $(3,4,5)$.

Failure of conservative treatment is one of the indications for surgical intervention. Recently, extracorporeal shock wave therapy (ESWT) has been advanced as a possible alternative to surgery in those patients recalcitrant to traditional conservative treatment $(6,7)$.

Shock wave therapy can be performed on an outpatient basis and, if effective, may prove to be cost-effective (1). A shock wave is a non-linear type of $15-20 \mathrm{Mhz}$ frequency pressure wave with an around $10 \mu$ rise time. These soundwaves define a positive and negative phase. The waves may hit an interface, in a first phase, with a reflection, or an absorption. In the tissue interface cavitation may occur, i.e. the formation of bubbles and their subsequent implosion with the genesis of a second wave (8). The propagation wave, through direct mechanical perturbation polarizing membrane cells, increases the density of the tissue and, 
therefore radical production, cell proliferation and growth factors formation (9). RSWT is an economic and noninvasive physical therapy with good clinical effects. In the rESWT device, a projectile is driven by compressed air or a magnetic field to impact onto the applicator at the head end of a handpiece for wave generation (9). Extracorporeal shock wave therapy (ESWT) had been proved to be beneficial in calcific tendinosis; however, the treatment efficacy in not calcific tendinosis of rotator cuff still remains controversial (10). The aim of this study is to present an evidence-based overview of the effectiveness of RSWT for the management of calcific tendinitis and non-calcific shoulder tendinopathy.

\section{METHODS}

The study was conducted ethically according to international standards (11).

\section{STUDY SELECTION}

Preferred Reporting Items for Systematic Reviews and Meta-Analyses guidelines were followed to conduct a systematic review. The research was conducted on PubMed, Embase, Physiotherapy Evidence Database (PEDro), Cochrane Controlled Trials Register, and the Cochrane databases by using the keywords: calcific tendinitis, ultrasound, shock wave, radial shockwave, RWST, shoulder, and rotator cuff and their expansions combined in algorithms, from the earliest record up to 30 June 2019. Electronic databases with English language restriction were searched for all relevant articles and included RTCs. Articles were initially screened by title and abstract. Articles unclear from title or abstract were reviewed according the selection criteria trough full text. Two authors independently extracted data from the studies that met the inclusion criteria and they were blinded to each other's (figure 1). A third and fourth opinion, was sought in case of disagreement.

\section{TYPES OF STUDIES}

RCTs were included where patients were randomly assigned to non-surgical treatments for calcific or non-calcific tendinitis of the shoulder or to control treatments versus sham or physiotherapy. Studies were excluded if the control

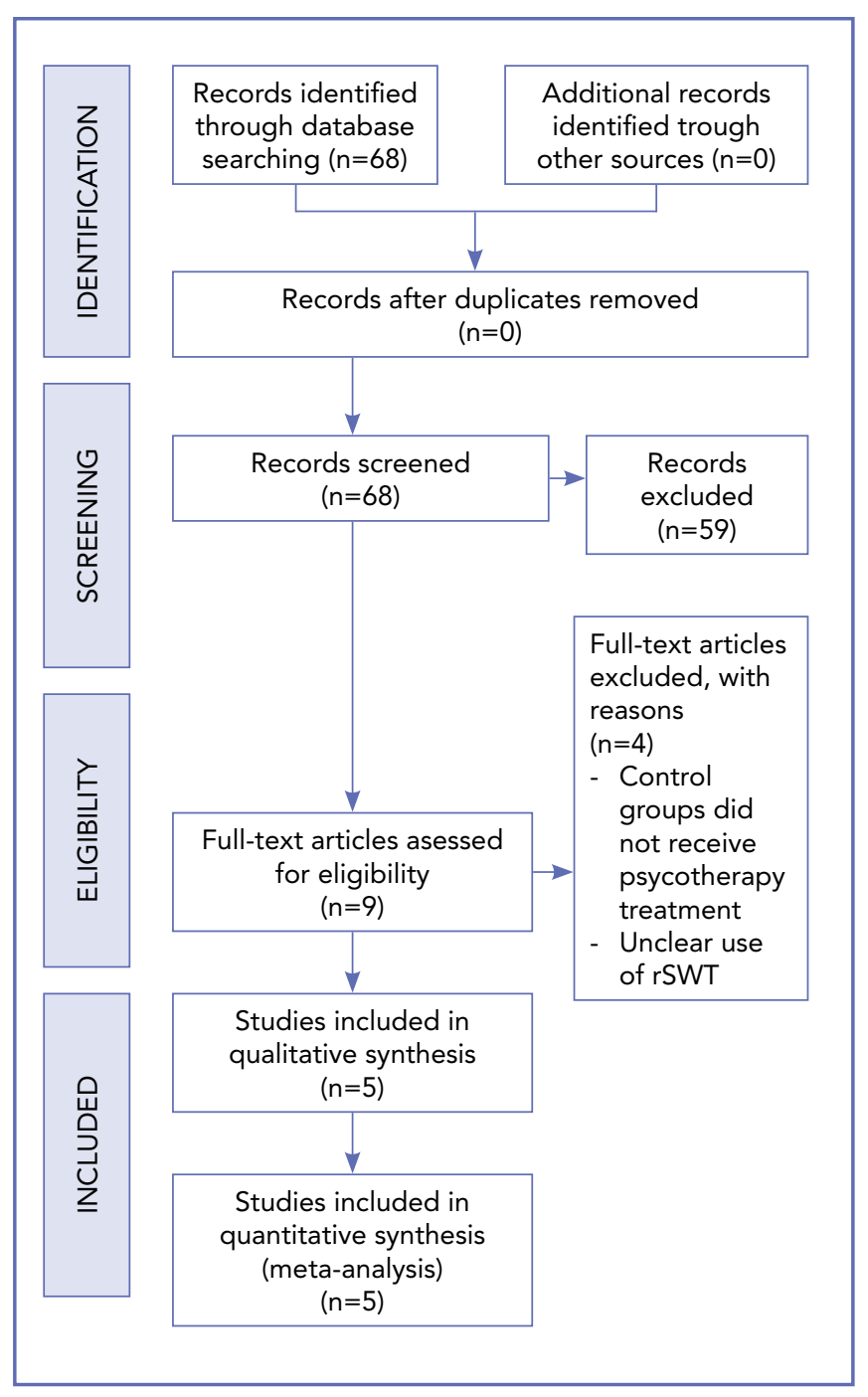

Figure 1. Flow diagram of randomized controlled trials evaluating the effectiveness of radial shockwave therapy in calcific and non-calcific tendinopathy of the shoulder tendinitis.

group received similar treatments and if outcome measures data could not be extracted or if the treatment site was not the shoulder. Studies were also excluded if patients were assigned to the same energy level of shockwave therapy or to different sections or a different total energy of shockwave therapy (table II).

Table I. Summary of included studies and patient characteristic.

\begin{tabular}{llll}
\hline Population & Intervention & Comparator & Outcome \\
\hline $\begin{array}{l}\text { Calcific and non-calcific } \\
\text { tendinopathy of the shoulder }\end{array}$ & $\begin{array}{l}\text { Radial ShockWave Therapy } \\
\text { (RSWT) }\end{array}$ & Traditional Physiotherapy & Pain and Shoulder Disability \\
\hline
\end{tabular}




\section{OUTCOMES}

The outcomes evaluated in our systemic review were improvement in pain severity and functional status of the shoulder. Shoulder pain was measured by means of the visual analog scale (VAS), in which pain intensity was rated from 0 (no pain) to 10 (worst pain). Disability assessment was measured by the shoulder pain and disability index (SPADI), a self-report questionnaire. The questionnaire consists of two domains: pain (five items) and disability (eight items). The total score ranges from 0 to 100 points, with a higher score indicating worse shoulder pain and disability (12). Duymaz performer the QuickDASH, an evaluation of the entire upper extremity function developed for musculoskeletal system disorders and scored in two components: the disability/symptom section (11 items) and the optional high-performance sport/music or work modules (four items). Each item is rated on a five-point Likert-type scale and the total cumulative score is scaled from 0 to 100 with higher scores indicating more disability (7). For functional assessment, the Constant-Murley score (CMS) and UCLA Shoulder rating scale were used. CMS consists of 4 components, including 25 points for shoulder muscle strength, 15 points for active, pain-free range of motion, 5 points for activities of daily living, and 15 points for pain. The maximum score (100 points) represents an asymptomatic patient. The UCLA Shoulder Rating Scale is a 35-point shoulder scale that combines scores for pain (score from 1 to 10), function (1-10), active range of forward flexion, strength of forward flexion, and patient satisfaction (all scored from 0 to 5 points). The outcome score is defined as follows: 34 to 35 points, excellent; 29 to 33 points, good; 21 to 28 points, mild; and 20 points or less, poor (13). The UCLA and Constant-Murley scores present high correlation (14). The pain score was extracted from CMS and adjustments were made to obtain data for pain severity if VAS data were not reported in the study. Data reported 18-24 months after treatment were used, and if a study did not report results measured at 18-24 weeks, we used those nearest to $18-24$ weeks.

\section{DATA EXTRACTION AND QUALITY MANAGEMENT}

Data extracted from the selected studies included: design, patient group (treatment / control), different treatment methods, average age (y), gender, duration of follow-up and outcome measurements. If there were missing information or any question about data accuracy, we contacted the authors of the original studies via e-mail to obtain further information. The guidelines of the Cochrane Handbook have been followed to assess the risk of bias. Study quality, such as selection bias, performance bias, detection bias, attrition bias, and selective reporting of results and other biases, was evaluated independently by 2 reviewers (figure 2). The differences between the extracted data and the quality assessment disagreements were discussed with a third and fourth author to reach a consensus.

\section{DATA SYNTHESIS AND ANALYSIS}

For pain reduction and functional improvement, data were expressed as the mean with SD. We calculated the weighted mean difference with $95 \%$ confidence intervals (CIs) between the baseline and status after treatment to pool continuous estimates of the result. Data were extracted at baseline and at the nearest 18-24 after treatment. First of all the data was extracted in a spreadsheet software for preliminary manipulations and then the statistical pack-

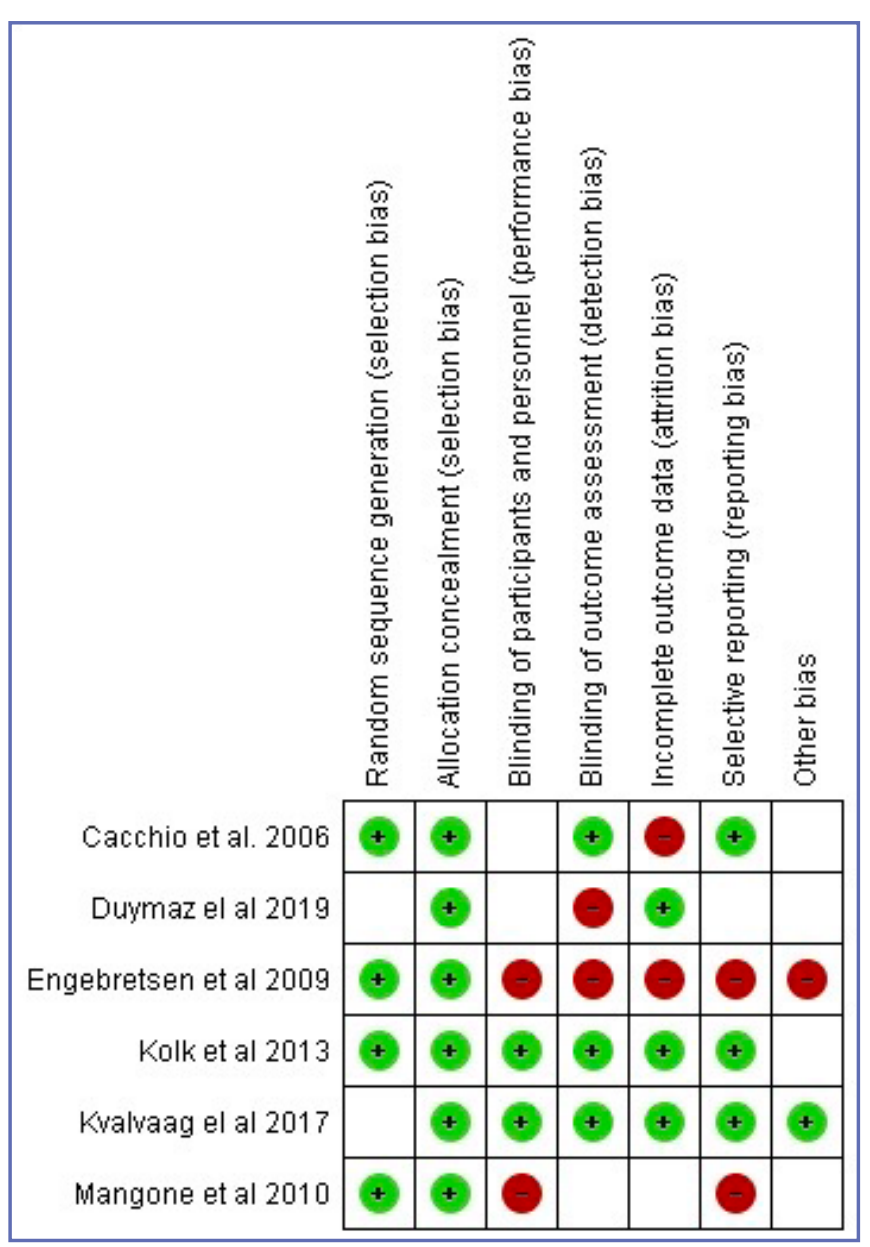

Figure 2. Summary of risk bias. 


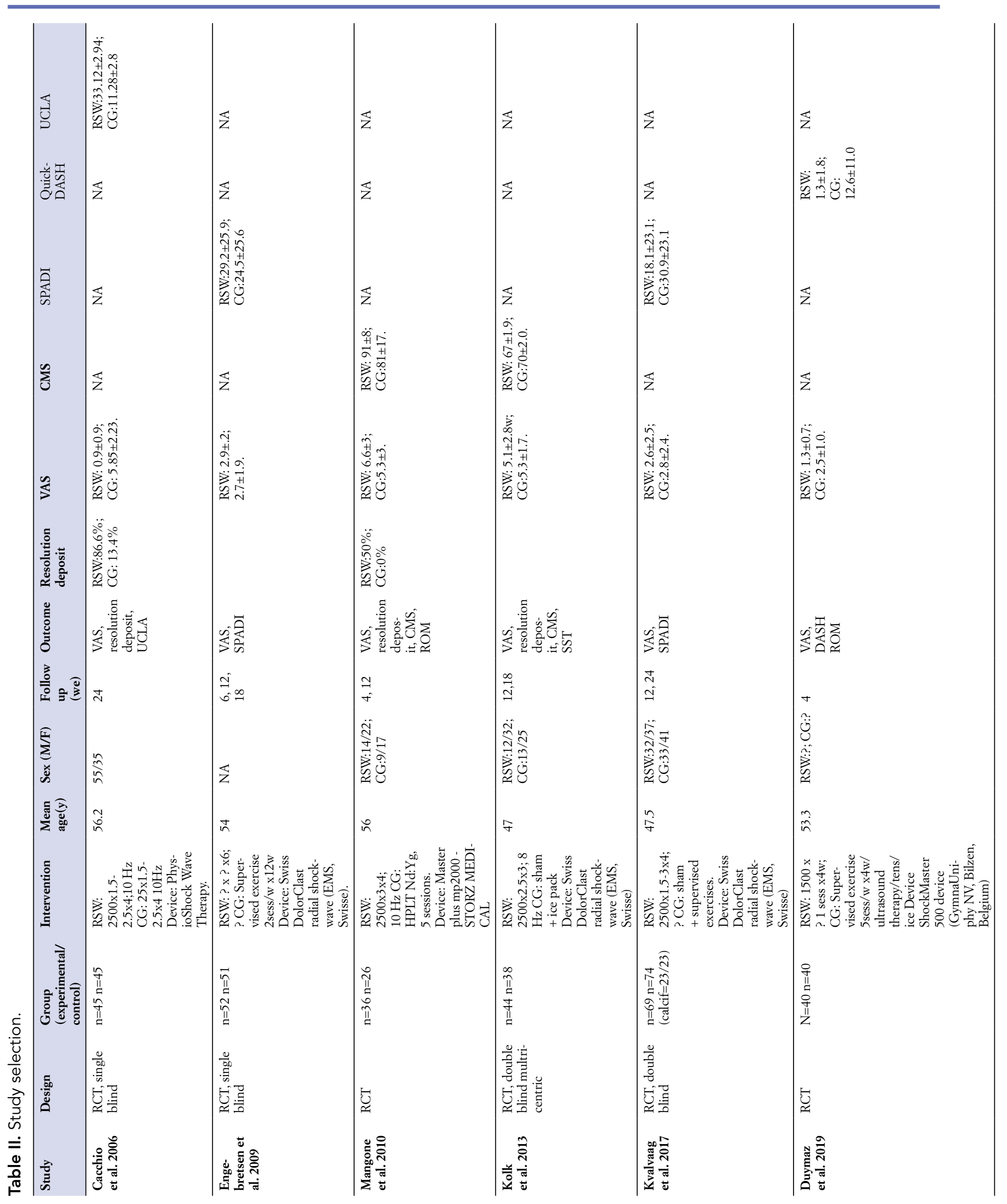


age in RevMan 5.3 (Cochrane Group - USA) was used for further analysis. A traditional meta-analysis was conducted for comparative tests.

\section{RESULTS}

A total of 68 articles, in the electronic databases, have been identified through the applied research strategy. 59 articles were excluded after reading the abstracts because they had no connection with the stipulated topic. Forty studies were not RCTs, did not use RSWT or its use was unclear. Of the nine potentially relevant articles read in full, four did not have a control group with a physiotherapy treatment and only five were included. In the research, all the studies initially presented similar groups regarding the most important prognostic indicators.

\section{SAMPLE CHARACTERISTICS}

The sample size of the surveys ranged from 36 (Mangone et al. (15)) to 69 individuals (Kvalvaag el al 2017 (16)). In the case of pain, the size of experimental group is 286 compared to a control group of 274 patients. The average age of the groups is $52(\mathrm{y})$.

As a diagnosis Cacchio et al. (13) used shoulder calcific/ non calcific tendinopathy, Mangone: calcific tendinosis of the rotator cuff, Kolk et al. (17) and Duymaz et al. (7): chronic rotator cuff tendinopathy of the shoulder, Engebretsen et al. (18) and Kvalvaag et al. (16): subacromial pain syndrome. Kvalvaag (16) considered a case/control subgroup $(n=23 / 23)$ with calcific disease.

\section{CHARACTERISTICS OF STUDY INTERVENTIONS}

Regarding the time of treatment with RSWT, the researchers showed a variability of 3 to 6 weeks, where 4 studies (Cacchio et al. (13), 2006; Mangone et al. (15), 2010; Kvalvaag et al. (16), 2017; Duymaz et al. (7)) observed the same period of 4 weeks. The number of shots was between 1500 and 2500 for session with a pressure ranged from 1.5 to 3 bar. Three studies reported a dummy control group (Cacchio et al. (13), Kolk et al. (17), E Kvalvaag et al. (16)); Engebretsen et al. (18) used supervised exercises; Mangone et al. (15) compared the RSWT treatment with 5 sessions of high-power laser therapy $(\mathrm{Nd}$ : Yag) as a control Group; Duymaz et al. (7) use as control traditional physiotherapy program include ultrasound (1.0 $\mathrm{MHz}, 5$ minutes, continuous), transcutaneous electrical nerve stimulation (conventional, 20 minutes), shoulder joint ROM and stretching exercises, and ice applications
(15 minutes). As regards the RSWT devices, Cacchio et al. (13) used PhysioShock ${ }^{\circledR}$ Wave Therapy (Pagani Elettronica, Milan, Italy); Mangone et al. (15) used Master Puls MP200 ${ }^{\circledR}$ (STORZ MEDICAL AG - Switzerland); Kolk et al (17), Engebretsen et al. (18) and Kvalvaag et al. (16) used Swiss DolorClast ${ }^{\circledR}$ (EMS Electro Medical Systems, Swiss); Duymaz et al. use ShockMaster 500 device (GymnaUniphy $\mathrm{NV}$, Bilzen, Belgium). Regarding the follow up period, the research showed a variability of 4 to 24 weeks, in 4 studies (Engebretsen et al. (18), Mangone et al. (15), Kolk et al. (17), Kvalvaag et al. (16)) was observed the same period of 12 weeks. Cacchio et al. (13) and Mangone et al. (15) used a frequency of shots of $10 \mathrm{~Hz}$, Kolk et al. (17) of $8 \mathrm{~Hz}$. The frequency of shots in Engebretsen et al. (18) and Kvalvaag et al. (16) unknown result.

\section{RESULT OF THE INTERVENTIONS}

The studies included in this review evaluated the pain and shoulder disability (figure 3 ).

Among the included studies that evaluated pain, through the Visual Analog Scale (VAS) or filled out another questionnaire, Cacchio et al. (13) and Duymaz et al. (7) reported a significant decrease $(0.9 \pm 0.9$ compared to $5.8 \pm 2.2 ; 1.7 \pm 0.7 / 2.5 \pm 1)$; Kolk et al. (17) and Kvalvaag et al. (16) showed a non-significant decrease (5.1 $\pm 2.6 / 5.3 \pm 2.7 ; 2.6 \pm 2.6 / 2.8 \pm 2.4)$; Engebretsen et al. (18) and Mangone et al. (15) reported an increase in pain $(2.9 \pm 2.1 / 2.6 \pm 1.9 ; 6.6 \pm 3 / 5.3 \pm 3)$.

Test for overall effect showed a significant decrease pain $(\mathrm{p}<0.05)$ in the experimental $(\mathrm{n}=286)$ compared to the control group $(\mathrm{n}=274)$. VAS score $=-1.14$ (95\% CI: -1.39 to $-0.89 \mathrm{Z}=8.84 \mathrm{p}<0.05)$

Analysis of CMS and UCLA shoulder function evaluations Cacchio et al. (13) and Mangone et al. (15) showed a significant improvement in the mean difference between groups, while Kolk et al. (17) did not report a significant difference in shoulder function.

The test for overall effect, combining the results of the CMS and UCLA scores, showed an improvement in experimental group $(n=125)$ compared to control $(n=109)$ in the functionality to satisfy the effectiveness in using the shoulder during the day living activities $(\mathrm{p}<0.05)$. Constant/UCLA score $=4.72$ (95\% CI: 4.03 to $5.4 \mathrm{Z}=13.5 \mathrm{p}<0.05)$.

Kvalvaag et al. (16) reported a decrease in shoulder disability score (SPADI) and Duymaz et al. (7) demonstrated an improvement in quick DASH evaluation in the experimental group compared to the control, while Engebretsen $e t$ al. (18) observed an increase in the disability index compared to the control group. The test for overall effect showed a significant reduction in shoulder pain and disability $(\mathrm{p}<0.05)$ in the experimental group $(n=161)$ compared to the control 


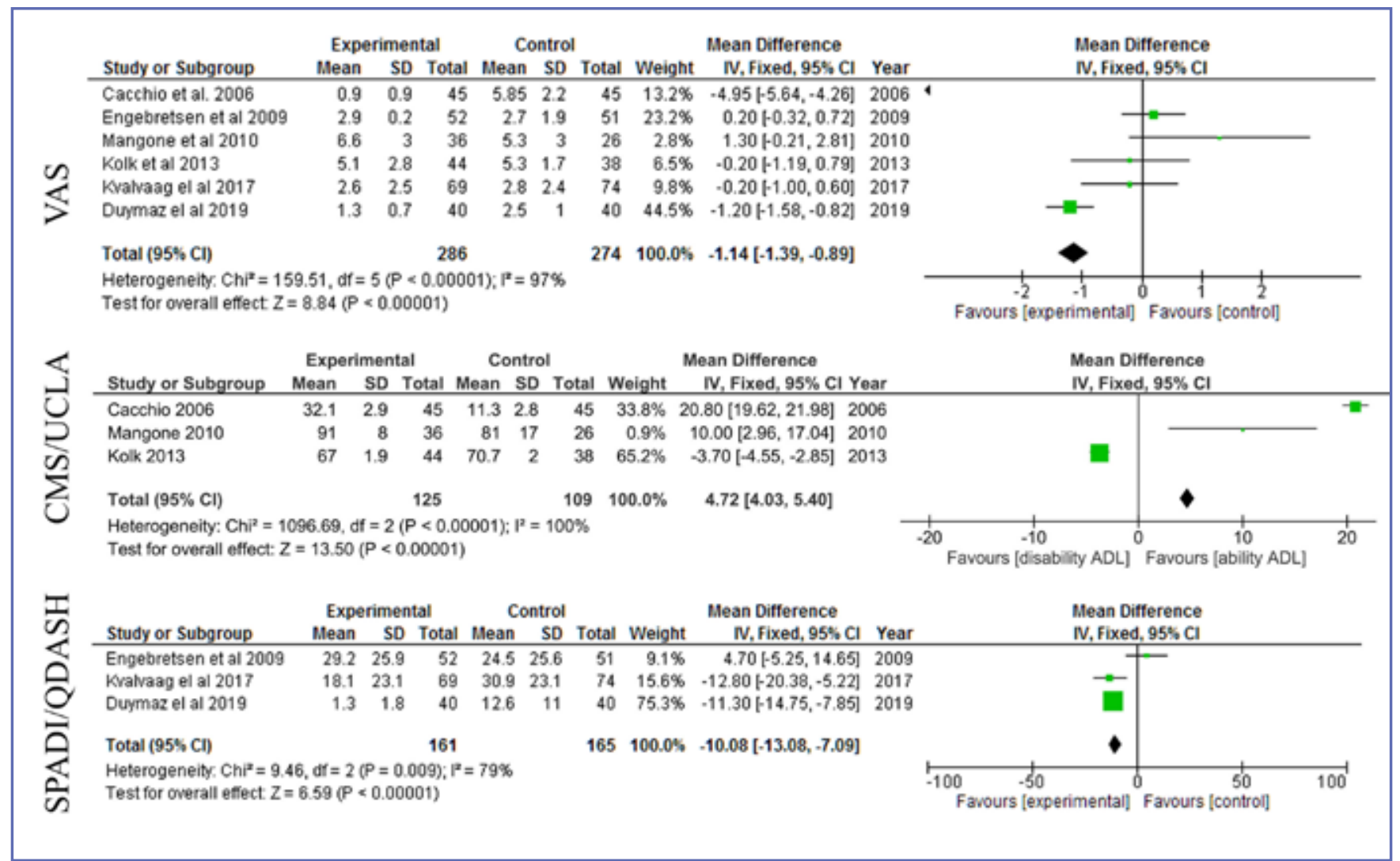

Figure 3. Forest plot of pain, shoulder functioning, shoulder disability. Abbreviation: VAS visual analogue scale, CMS Constant-Murley scale, SPADI shoulder pain and disability index.

group ( $\mathrm{n}=165)$. SPADI/quick $\mathrm{DASH}=-10.76(95 \% \mathrm{CI}$ : -13.08 to $-7.09 ; \mathrm{Z}=6.59 \mathrm{p}<0.05)$.

\section{DISCUSSION}

Shoulder pain is one of the most common musculoskeletal pathologies. ESWT showed beneficial effects on calcific tendinopathy of the shoulder and quality of life over a 2 years follow-up period (19). Treatment by ESWT has considered as valid alternative in rotator cuff calcific tendinopathy, while the clinical efficacy in non-calcific tendinopathy remains controversial. The rapid growth of RSWT in recent years has positioned it as an alternative to focal ESWT for the treatment for rotator cuff calcifications, but its role in non-calcific tendinitis is unclear (20). Unlike focal shock waves, RSWT have physical properties significantly different: linear pressure, low energy values, relatively low velocity of propagation and short duration of the rise time (21). While the focal shock waves have an anti-inflammatory effect, because they are able to change of concentration of flogosis mediators (22), RSWT allow to obtain a reduction of pain with improvement of joint function, through mechanisms not yet fully known (23). This systematic review and meta-analysis of six RCTs demonstrated that RSWT was effective in decreasing pain and improve quality of life, compared to sham, supervised or physical treatment.

Test for overall effect showed a significant decrease pain $(\mathrm{p}<0.05)$ in the experimental compared to the control group. Engebretsen et al. (18) showed not significant difference in VAS score, but Engebretsen et al. (18) compared RSWT and supervised exercises plus sham-RSWT, so the absence of significant difference could be due to the effectiveness of the exercises in the treatment of subacromial impingement syndrome (24) Mangone et al. (15) compared RSWT and High-Power LASER Therapy (HPLT), the difference in pain score is not significant at the end of the treatment, but becomes significant one and three months after.

Kolk et al. (17) reported non-significant difference of the relief of pain in experimental group compared to control group, that might be several reasons for these results, such as the relevant number of variables in the application of RSWT: energy flux density, sessions and shots for session, 
frequency, etc. (25). In agreement to other authors, Kvalvaag et al. (16) distinguished the non-calcific from the calcific and underlined that the improvement in the calcified is better (20).

The test for overall effect, combining the results of the CMS and UCLA scores, showed an improvement of the shoulder joint function during the day living activities $(\mathrm{p}<0.05)$. The same result is reported for the evaluation of the SPADI score Kvalvaag et al. (16) $(\mathrm{p}<0.05)$. Cacchio et al. (13) demonstrated an increase in UCLA score and Mangone et in CMS score. Kolk et al. (17) reported similar improvement of CMS in both groups of participants (treatment and placebo) without significant difference between the groups. Only Engebretsen et al. (18) observed a worsening of the function of the shoulder joint in the group treated by RSWT compared to the control group treated by supervised exercises, this could be due to the important difference in the duration of the treatments: 12 weeks for exercises against 4 sessions of RSWT.

Cacchio et al. (13) also reported a complete disappearance of calcific deposits in $86 \%$ of individuals and partial reabsorption in 13,4\%. Mangone et al. (15) observed the disappearance of calcification in 16 shoulders $(31 \%)$ and the decrease of calcification in 10 shoulders (19\%).

\section{STUDY LIMITATIONS}

There are several limitations to this study. Only 5 randomized controlled trials were included. The function and disability of the shoulder is an important outcome, but only 3 and 2 randomized controlled trials were analyzed respectively for functional assessment and disability in the meta-analysis. Cacchio et al. (13) used the University of California, Los Angeles Shoulder Rating Scale to evaluate the effectiveness of RSW in repairing shoulder function. Engebretsen et al. (18), did not clearly describe the parameters used by the device.

\section{CONCLUSIONS}

This review confirms the effectiveness of the RSWT in the treatment of the calcific and non-calcific tendinopathy of the shoulder, but better results are obtained in calcific tendinopathy, in which it is possible to observe the disappearance of the calcification in over $50 \%$ of the cases. All trials were randomized and were deemed qualify and provided estimate of the treatment effects and used intention-to-treat analysis, which is an essential feature of an RCT. However, further studies are needed to standardize parameters to apply RSWT, such as: energy flux density, number of shots and sessions, frequency of the shots.

\section{CONFLICT OF INTERESTS}

The authors declare that they have no conflict of interests.

\section{REFERENCES}

1. Harniman, E., Carette, S., Kennedy, C., \& Beaton, D. (2004). Extracorporeal shock wave therapy for calcific and noncalcific tendonitis of the rotator cuff: a systematic review. Journal of Hand Therapy, 17(2), 132-151.

2. Hurt G, Baker CL Jr. Calcific tendinitis of the shoulder Orthop Clin North Am 2003; 34:567-75.

3. Elsodany AM, Alayat MSM, Ali MME, Khaprani HM. LongTerm Effect of Pulsed Nd:YAG Laser in the Treatment of Patients with Rotator Cuff Tendinopathy: A Randomized Controlled Trial. Photomed Laser Surg. 2018 Sep;36(9):506-513.

4. Heron SR, Woby SR, Thompson DP. Comparison of three types of exercise in the treatment of rotator cuff tendinopathy/ shoulder impingement syndrome: A randomized controlled trial., Physiotherapy. 2017;103(2):167-173.

5. Fusaro I, Orsini S, Diani S, Saffioti G, Zaccarelli L, Galletti S. Functional results in calcific tendinitis of the shoulder treated with the rehabilitation after ultrasonic-guided approach. Musculoskelet Surg 2011; 95 (Suppl 1):S 31-6.

6. Vestraelen FU, In den Kleef NJ, Jansen L, Morrenhof JW. High-energy versus low-energy extracorporeal shock wave therapy for calcifying tendinitis of the shoulder: which is superior? A Metanalysis. Clin Orhop Relat Res 2014; 472: 2816-25.

7. Duymaz T, Sindel D. Comparison of Radial Extracorporeal Shock Wave Therapy and Traditional Physiotherapy in Rotator Cuff Calcific Tendinitis Treatment. Arch Rheumatol. 2019 Jan 28;34(3):281-287.

8. Ogden, J. A., Tóth-Kischkat, A., \& Schultheiss, R. (2001). Principles of shock wave therapy. Clinical Orthopaedics and Related Research (1976-2007), 387, 8-17.

9. Rosso, F., Bonasia, D. E., Marmotti, A., Cottino, U., \& Rossi, R. 2015. Mechanical stimulation (pulsed electromagnetic fields "PEMF" and extracorporeal shock wave therapy "ESWT") and tendon regeneration: a possible alternative., Frontiers in aging neuroscience, $7,211$.

10. Efficacy of Extracorporeal Shockwave Therapy on Calcified and Noncalcified Shoulder Tendinosis: A Propensity Score Matched Analysis. Wu KT, Chou WY, Wang CJ, Chen CY, Ko JY, Chen PC, Cheng JH, Yang YJ. Biomed Res Int. 2019 Mar 14;2019:2958251.

11. Padulo J., Oliva F., Frizziero A., Maffulli N. Muscles, Ligaments and Tendons Journal - Basic principles and recommendations in clinical and field Science Research: 2018 update. MLTJ 2018; 8(3): 305 - 307.

12. Roach KE, Budiman-Mak E, Songsiridej N, Lertratanakul Y. Development of a shoulder pain and disability index. Arthritis Care Res, 1991;4:143-9.

13. Cacchio, Angelo, et al. "Effectiveness of radial shock-wave therapy for calcific tendinitis of the shoulder: single-blind, randomized clinical study." Physical therapy 86.5 2006: 672-682.

14. Malavolta, Eduardo \& Assunção, Jorge \& Gracitelli, Mauro \& Antonio Araújo Simões, Pedro \& Kenji Shido, Danilo \& Neto, Arnaldo. 2018) Correlation between the UCLA and 
Constant-Murley scores in rotator cuff repairs and proximal humeral fractures, osteosynthesis. Revista Brasileira de Ortopedia (English Edition). 53.

15. Mangone, G., Veliaj, A., Postiglione, M., Viliani, T., \& Pasquetti, P. 2010. Radial extracorporeal shock-wave therapy in rotator cuff calcific tendinosis. Clinical cases in mineral and bone metabolism, 7(2), 91.

16. Kvalvaag, E., Brox, J. I., Engebretsen, K. B., Soberg, H. L., Juel, N. G., Bautz-Holter, E., ... \& Roe, C. 2017. Effectiveness of radial extracorporeal shock wave therapy (rESWT) when, combined with supervised exercises in patients with subacromial shoulder pain: a double-masked, randomized, sham-controlled trial. The American journal of sports medicine, 45(11), 2547-2554.

17. Kolk, A., Auw Yang, K. G., Tamminga, R., \& Van Der Hoev-en, H. 2013. Radial extracorporeal shock-wave therapy in patients with chronic rotator cuff tendinitis: a prospective randomised double-blind placebo-controlled multicentre trial. The bone \&, joint journal, 95(11),

18. Engrebrezsen, K., Grotle, M., Bautz-Holter, E., Sandvik, L., Juel, N. G., Ekeberg, O. M., \& Brox, J. I. 2009. Radial extracorporeal shockwave treatment compared with supervised exercises in patients with subacromial pain syndrome: single blind randomised, study. Bmj, 339, b3360.

19. Maffulli, G., Iuliano, E., Padulo, J., Furia, J., Rompe, J., \& Maffulli, N. 2018. Extracorporeal shock wave therapy in the treatment of calcific tendinopathy of the shoulder: the ASSERT database. Muscles, Ligaments \& Tendons Journal (MLTJ), 8(3).

20. Huisstede BM, Gebremariam L, van der Sande R, Hay EM, Koes BW. Evidence for effectiveness of Extracorporal ShockWave Therapy (ESWT) to treat calcific and non-calcific rota- tor cuff tendinosis--a systematic review. Man Ther. 2011 Oct;16(5):419-33.

21. Van der Worp H, Van den Akker S, Van Schie H, et al. ESWT for tendinopathy: technology and clinical implications. Knee Surg Sports Traumatol Arthrosc. 2012;21:1451-1458.

22. M. B. Notarnicola A, The biological effects of extracorporeal shock wave therapy (eswt) on tendon tissue., Muscles Ligaments Tendons J. 2012 Jun 17;2(1):33-7.

23. Friso A. De Boer, Femke Mocking, Eelco M. Nelissen, Paulien M. Van Kampen, Pol E. Huijsmansa Ultrasound guided Needling vs Radial Shockwave Therapy in calcific tendinitis of the shoulder: A prospective randomized trial, J Orthop. 2017 Dec; 14(4): 466-469.

24. Vinuesa-Montoya S, Aguilar-Ferrándiz ME, Matarán-Peñarrocha GA, Fernández-Sánchez M, Fernández-Espinar EM, Castro-Sánchez AM. A Preliminary Randomized Clinical Trial on the Effect of Cervicothoracic Manipulation, Plus Supervised Exercises vs a Home Exercise Program for the Treatment of Shoulder Impingement. J Chiropr Med. 2017 Jun;16(2):85-93.

25. Ioppolo F, Rompe JD, Furia JP, Cacchio A. Clinical application of shock wave therapy (SWT) in musculoskeletal disorders. Eur J Phys Rehabil Med. 2014 Apr;50(2):217-30.

26. Wu, Y. C., Tsai, W. C., Tu, Y. K., \& Yu, T. Y. (2017). Comparative effectiveness of nonoperative treatments for chronic calcific tendinitis of the shoulder: a systematic review and network meta-analysis of randomized controlled trials., Archives of physical medicine and rehabilitation, 98(8), 1678-1692.

27. Van der Worp, H., van den Akker-Scheek, I., van Schie, H., \& Zwerver, J. 2013. ESWT for tendinopathy: technology and clinical implications. Knee Surgery, Sports Traumatology, Arthroscopy, 21(6), 1451-1458.

28. Hurt G, Baker CL Jr. Calcific tendinitis of the shoulder Orthop Clin North Am 2003; 34:567-75. 